\title{
Obtaining a Rough Flux Front in Type-II Superconductors Using a Critical State Model
}

\author{
C. Romero-Salazar ${ }^{a, *}$, O.A. Hernández-Flores ${ }^{a}$, V. Chabanenko ${ }^{b}$, E.I. Kuchuk ${ }^{b}$, \\ I. Abaloszewa ${ }^{c}, A$. NABialeK ${ }^{c}$ And F. PÉRez-Rodríguez ${ }^{d}$ \\ ${ }^{a}$ Escuela de Ciencias, Universidad Autónoma "Benito Juárez" de Oaxaca, Oaxaca 68120, Mexico \\ ${ }^{b}$ Galkin Donetsk Institute for Physics and Engineering, NAS, Kyiv 03668, Ukraine \\ ${ }^{c}$ Institute of Physics, Polish Academy of Sciences, Aleja Lotnikow 32/46, PL-02668 Warsaw, Poland \\ ${ }^{d}$ Instituto de Física, Benemérita Universidad Autónoma de Puebla, \\ Apartado Postal J-48, Puebla, Pue. 72570, Mexico
}

\begin{abstract}
In this work we numerically modelled a periodic magnetic flux pattern which qualitatively reproduces the so-called sand avalanches scenario in type-II superconductors. To model these sand-pile patterns we consider a perturbation on the critical current which, as a first approximation, follows a periodic function which depends on the position.
\end{abstract}

DOI: 10.12693/APhysPolA.130.645

PACS/topics: 74.20.-z, 74.20.De, 74.25.Ha, 74.25.N-, 74.25.Op, 74.25.Sv, 74.25.Wx, 74.62.Dh, 74.81.-g

\section{Introduction}

The magneto-optical technique has revealed a stochastic distribution of the magnetic flux in type-II superconducting films, therein, a rough penetration front is formed when a stationary state is reached. The roughness depends on the temperature because at the threshold temperature $T_{\mathrm{sp}}$ (subscript "sp" means a sand pile scenario) the landscape looks like sand avalanches distributed randomly throughout the border sample [1, 2]; on the other hand, at temperatures $T_{\mathrm{ca}}<T_{\mathrm{sp}}$ (subscript "ca" indicates a catastrophic avalanche regime) the scenario is a consequence of dendrites nucleation [3].

According to our magneto-optical images (see Fig. 1) obtained at the surface of the $\mathrm{NbTi}$ at $50 \%$, a material widely used in technology, the magnetic profiles have a "rocky-mountain-like" slope due to stochastic jumps of the flux bundles which form a rough flux front [1]. The influence of such non-uniform flux front relief map may be significant on the upper shielding limit of the full critical state stability.

The characterization of the magnetic instabilities at these different temperatures regions is useful for technological applications and fundamental studies. Specifically, changes in perturbations such as the applied magnetic field or the local temperature are of great interest, for this purpose, the stability condition of the critical state and the magnetic induction behaviour at each region, is required.

Our goal is to model the magnetic induction at regions where the sand-pile scenario occurs, so in this work we theoretically model a periodic flux pattern which quali-

\footnotetext{
*corresponding author; e-mail: cromeros@ifuap.buap.mx
}

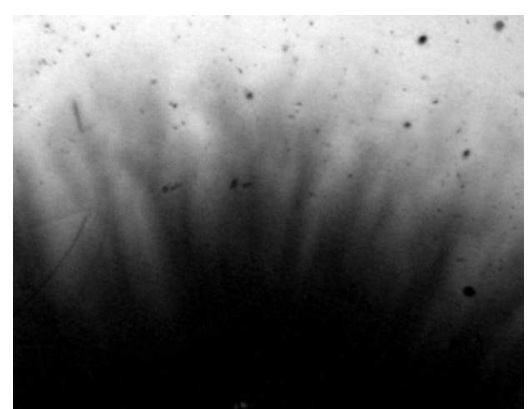

Fig. 1. Magneto-optical images of flux penetration in external magnetic field $H_{a}=400 \mathrm{G}$ in a NbTi disc with diameter $12 \mathrm{~mm} ; T=6 \mathrm{~K}$.

tatively reproduces the scenario of sand avalanches [1, 2]. This kind of flux pattern is important as a trigger, first, for large catastrophic thermomagnetic avalanches and, second, for the scenario of dendrites flux. To model flux patterns as the so-called sand-pile type we consider a perturbation on the critical current which, as first approximation, is a periodic function depending on the position [4]. In the next section, we show the modelling of flux patterns, coming up next we present graphics of flux distribution for an infinite plate and we finish with our conclusions.

\section{Modelling rough flux fronts with a perturbed critical current density}

Let us consider a semi-infinite superconducting plate having its surface on the $y z$-plane and being under an external magnetic field $\boldsymbol{H}_{a}=H_{a} \hat{z}$. For this geometry, the magnetic field $\boldsymbol{H}$, the magnetic induction $\boldsymbol{B}$, the current density $\boldsymbol{j}$ and the electric field $\boldsymbol{E}$, are independent of the 
variable $z$. This study starts at the scenario where there is a sand-pile landscape which originates deviations of the current density (see parts (A) and (B) of Fig. 2). Also, we assume the existence of nucleation sites of avalanches periodically distributed at the borders, and that the flux fronts have a periodic behaviour as it is shown schematically at part (C) of Fig. 2. These considerations lead to the electromagnetic fields that depend on the variables $x$ and $y$, thus in our macroscopic approach the Ampere and Faraday equations are written as

$$
\begin{aligned}
& \mu_{0} j_{x}=\partial_{y} B_{z}, \quad \mu_{0} j_{y}=-\partial_{x} B_{z}, \\
& \partial_{t} B_{z}=-\partial_{x} E_{y}+\partial_{y} E_{x} .
\end{aligned}
$$
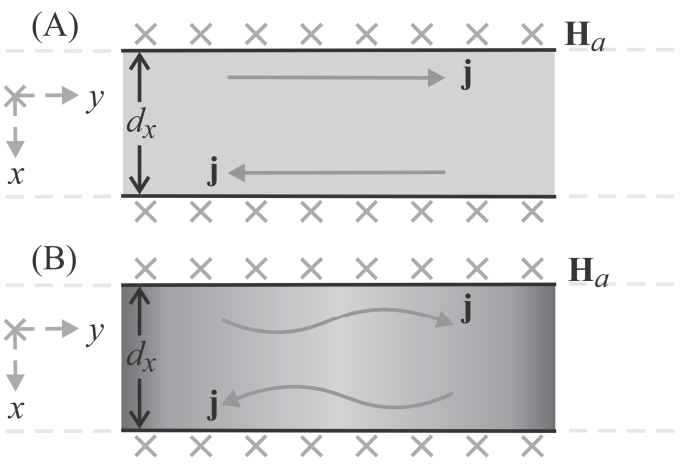

(C)

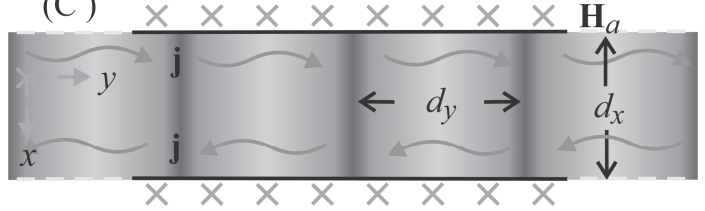

Fig. 2. Sketch of the $x y$-plane of an infinite plate under $\boldsymbol{H}_{a}=H_{a} \hat{z}$. The current density $\boldsymbol{j}$ suffers a periodic perturbation along the $y$-direction.

Since the effects of surface barrier are neglected, the boundary condition $B_{z}(0, y)=B_{z}\left(d_{x}, y\right)=\mu_{0} H_{a}$ is fulfilled, and due to the imposed periodicity it is considered that $B_{z}(x, y)=B_{z}\left(x, y+d_{y}\right)$. To solve the Maxwell equations we include the superconducting character of the system through the material law

$$
j_{i}=J_{i k} \frac{E_{k}}{E},
$$

where $J_{i k}=J_{i} \delta_{i k}(i, k=x, y)$ are the elements of a diagonal tensor. Once the critical state is established, the tensor components are $J_{x x}=j_{c x}$ and $J_{y y}=j_{c y}$, so we obtain from material law (2) the relations $E_{x}=E j_{x} / j_{c x}$ and $E_{y}=E j_{y} / j_{c y}$. The magnitude of the electric field is modelled with a vertical law $E=\mathbb{H}\left(j-j_{c}\right) \rho\left(j-j_{c}\right)$, where the resistivity $\rho$ plays the role of an auxiliary parameter, and $\mathbb{H}\left(j-j_{c}\right)$ is the Heaviside function. Additionally, with $\boldsymbol{E} \cdot \boldsymbol{E}=E^{2}$ it was found that the magnitude of the critical current is

$$
\frac{1}{j_{c}^{2}}=\frac{\cos ^{2} \phi}{j_{c x}^{2}}+\frac{\sin ^{2} \phi}{j_{c y}^{2}},
$$

where $\phi$ is the angle of the critical current density with respect to the $x$-axis. Every point of the ellipse describes a particular critical state; this critical ellipse is the heart of the elliptic critical state models [5]. The ellipse path is the restriction which provides us with certainty of being in a critical state. Geometrically speaking, the critical state corresponds to the intersection of the ellipse with the circumference described by the Euclidean distance $|\boldsymbol{j}|=j_{c}$.

Our model consists in adding a perturbation to a reference value of the critical current density $j_{c y}$, that is, $j_{c x}=j_{c x}^{K A}$ and $j_{c y}=j_{c y}^{K A}+\Delta j_{c y}$ where $j_{c x}^{K A}$ and $j_{c y}^{K A}$ obeys the generalized Kim-Anderson function

$$
j_{c x}^{K A}=\frac{j_{0 x}}{\left(1+B / B_{x}^{*}\right)^{n_{x}}}, \quad j_{c y}^{K A}=\frac{j_{0 y}}{\left(1+B / B_{y}^{*}\right)^{n_{y}}} .
$$

Here $j_{c x}(0)=j_{0 x}, j_{c y}(0)=j_{0 y}, B_{x}^{*}, B_{y}^{*}, n_{x}$ and $n_{y}$ are fitting parameters. If the material is isotropic at the plane $x y$, then $j_{c x}^{K A}=j_{c y}^{K A}$, thus the disturbance leads to an apparent spatial anisotropy produced by the nucleation of avalanches. Moreover, the bending of the $\boldsymbol{j}$ trajectory gives rise to a dependence on the $x$-component. It is assumed that $\Delta j_{c y}$ is a periodic function given by $\Delta j_{c y}=j_{0 y}\left(B / B_{\Delta y}\right) \cos (\alpha y+\beta)$ where $B_{\Delta y}$ is a constant, $\beta$ is a phase, $\alpha=n \pi / d_{y}, n$ is an even integer and $d_{y}$ is the spatial period. Finally, we establish that the set of Eqs. (1)-(4) depict an unusual critical state since an artificial anisotropy, associated to the avalanches nucleation at the edges of the sample, has been considered.

\section{Results and discussion}

To numerically solve the quasi-stationary Maxwell equations - together with the material law and the KimAnderson relations - we use the method of lines [6] mediated by the critical ellipse. We considered a YBCO sample of thickness $d_{x}=3 \times 10^{-4} \mathrm{~m}$ and penetration field $\mu_{0} H_{p}=8.565 \times 10^{-2} \mathrm{~T}$. The external field is applied perpendicular to the isotropic crystallographic plane of the sample, therefore, the relation $j_{c x}^{K A}=j_{c y}^{K A}$ is fulfilled and the fitting parameters are $n_{x}=n_{y}=0.5$, $B_{x}^{*}=B_{y}^{*}=0.1 \mathrm{~T}$ and $j_{0 x}=j_{0 y}$. The spatial period matches with the thickness $d_{y}=d_{x}$.

We emulated rough flux fronts as can be appreciated in Figs. 3 and 4, considering that the system is at an unusual critical state, created by a flux distribution which has been deformed by magnetic induction jets emerging from the border sample. We artificially created the rough flux front imposing an anisotropy on the $x-y$ plane.

In order to understand better the flux front behaviour, we first focus on the case of a $B_{z}(x, y)$ distribution with one oscillation at one spatial period. Thus, in Fig. 5 we show a gray color map of the magnetic induction corresponding to the unperturbed case when the applied field reaches the value $\boldsymbol{H}_{a}=\left(H_{p} / 2\right) \hat{z}$. For this case the right scale denotes the values of $B_{z} / \mu_{0} H_{p}$ and the horizontal white line corresponds to the profile $B_{z}=0.1 \mu_{0} H_{p}$. We superimposed over the gray map another two profiles at the same level $B_{z}=0.1 \mu_{0} H_{p}$ but for different values of the perturbation amplitude $B_{\Delta y}=0.5,0.1 \mu_{0} H_{p}$ to show 


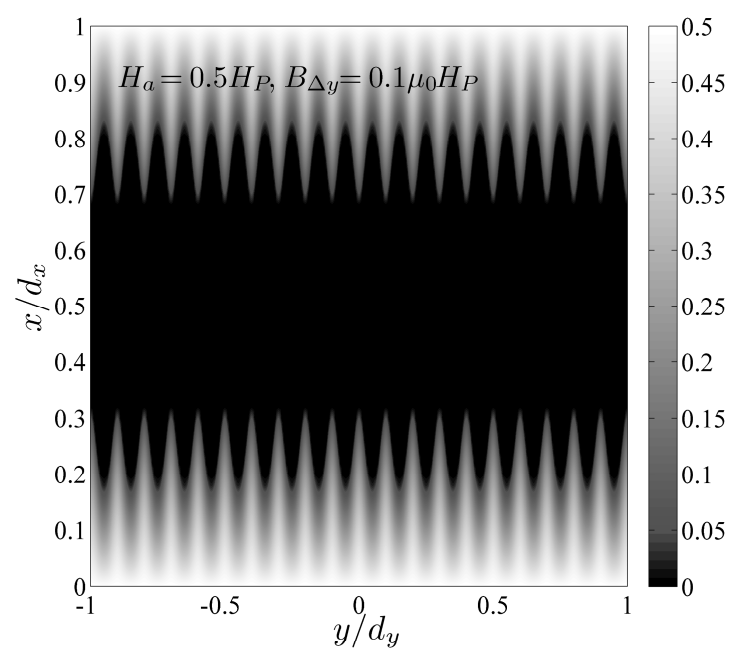

Fig. 3. Color maps of $B_{z}(x, y)$ for $H_{a}=0.5 H_{p}, B_{\Delta y}=$ $0.1 \mu_{0} H_{p}$, two spatial periods and ten oscillations.

how they are deviated from the unperturbed case. We have considered a single one oscillation, $\alpha=2 \pi / d_{y}$, with phase $\beta=\pi$, to make clear the perturbation effect on the profiles. As is expected, the perturbed magnetic induction distribution exhibits a periodic behaviour but each profile does not oscillate around the unperturbed one because the perturbation linearly depends on the magnetic induction. Let us observe the perturbed profiles, when $\Delta j_{y}$ is positive, the $\boldsymbol{B}$ penetration is promoted, otherwise, such a penetration is inhibited. This behaviour is a consequence of the elliptic model which couples the critical current densities $j_{c x}$ and $j_{c y}$, where only the later suffers small changes.

One can observe in Fig. 3 the $B_{z}(x, y)$ distributions for partial penetration states at an applied field $H_{a}=0.5 H_{p}$, a perturbation $B_{\Delta y}=0.5 \mu_{0} H_{p}$ and two spatial periods. They bear some resemblance to a box pencil drawing, the upper gray map is obtained with five oscillations and the bottom one has ten oscillations. Figure 3 shows the effect of the perturbation amplitude, with $B_{\Delta y}=0.1 \mu_{0} H_{p}$ a deeper penetration of the flux profiles is achieved. Notice that those figures are not spatially proportional, therefore the profiles in Fig. 3 looks sharper than those of Fig. 4.

\section{Conclusion}

The emulation of flux pattern into the sand-pile scenario was successful for an infinite superconducting plate under an external magnetic field at the parallel geometry. These rough profiles can be employed as initial magnetic states to study the effect of thermomagnetic instability.

Indeed, modelling real materials behaviour through more irregular flux patterns than a periodic one is numerically challenging. However, periodic flux fronts as
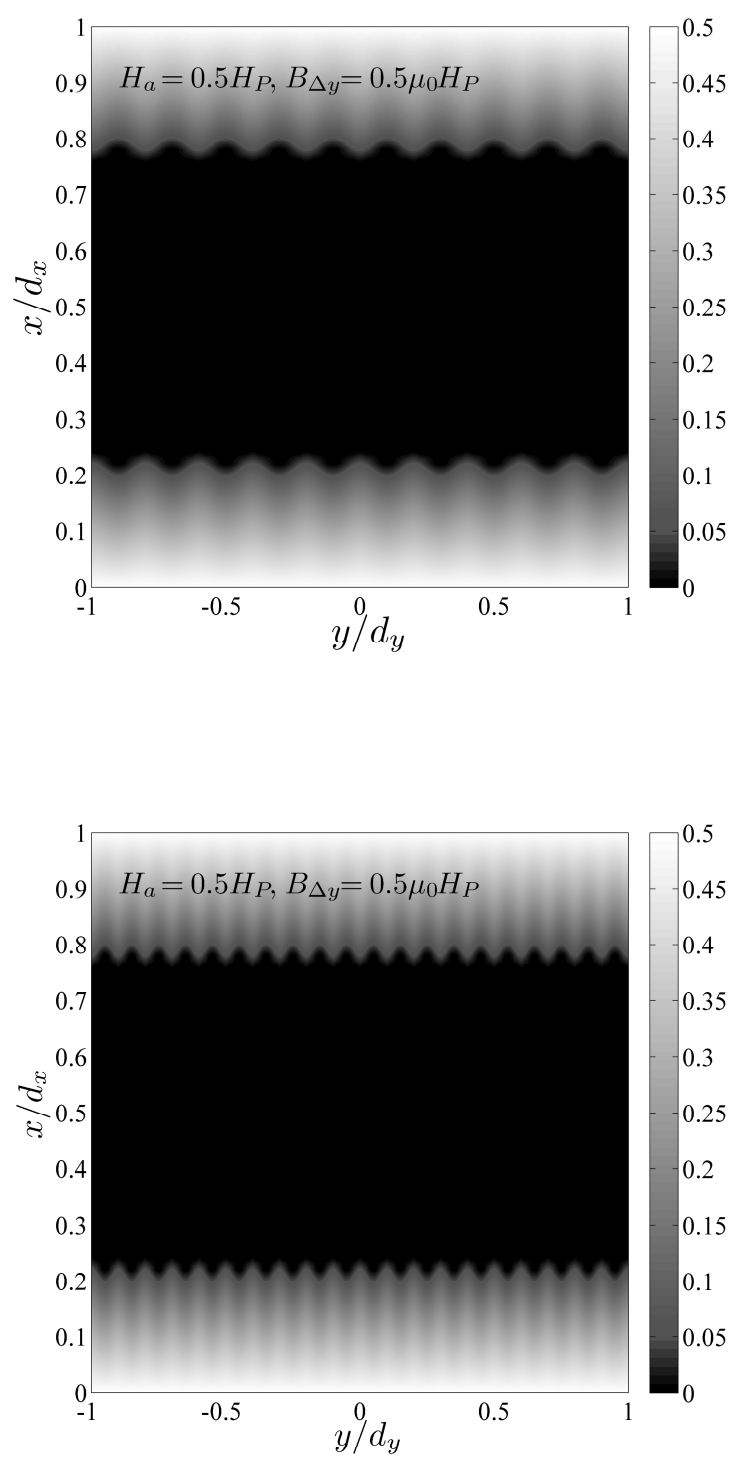

Fig. 4. Color maps of $B_{z}(x, y)$ for $H_{a}=0.5 H_{p}, B_{\Delta y}=$ $0.5 \mu_{0} H_{p}$ and two spatial periods. The upper gray map is obtained with five oscillations, the bottom gray map has ten oscillations.

those presented in this paper can be useful to study certain phenomena at the so-called catastrophic magnetic flux avalanches regime, where temperature plays an important role. Even so, flux fronts with certain irregularities can be modelled at the infinite parallel geometry; the only requirement is that they fulfil periodic boundary conditions. In other words, each rough flux front should exist in a unit cell to be reproduced infinitely along the $y$-direction. It is possible to model a randomly irregular flux-front profile by modulating the periodic perturbation with a random amplitude, however, we cons ider that the global behaviour of the thermomagnetic instabilities does not require this kind of precursory profile. 


\section{Acknowledgments}

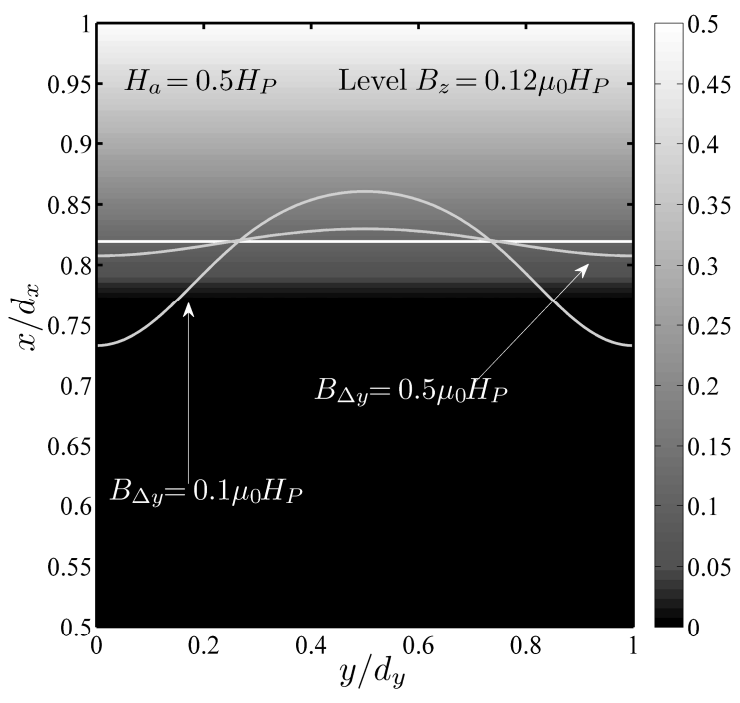

Fig. 5. The applied magnetic field is $\boldsymbol{H}_{a}=\left(H_{p} / 2\right) \hat{z}$ and the current density $\boldsymbol{j}$ is perturbed along the $y$ direction as $\Delta j_{c y}=j_{0 y}\left(B / B_{\Delta y}\right) \cos (\alpha y+\beta)$. The gray scale denotes the values of $B_{z} / \mu_{0} H_{p}$, the gray map corresponds to the unperturbed case and the white horizontal line to the profile $B_{z}=0.1 B_{p}$; there are superimposed over the gray map two profiles with the same value $B_{z}=0.1 B_{p}$ and amplitudes $B_{\Delta y}=0.5,0.1 \mu_{0} H_{p}$ to exhibit how they deviate from the unperturbed case. It is assumed that $\alpha=2 \pi / d_{y}$ and $\beta=\pi$.
C. Romero-Salazar acknowledges support by UABJO (Mexico) under grant PROFOCIE-2014. F. PérezRodríguez acknowledges support by SEP-CONACYT (Mexico) under grant CB-2012-01-183673, and by VIEPBUAP.

\section{References}

[1] V.K. Vlasko-Vlasov, U. Welp, V. Metlushko, G.W. Crabtree, Phys. Rev. B 69, 140504(R) (2004).

[2] R. Surdeanu, R.J. Wijngaarden, E. Visser, J.M. Huijbregtse, J.H. Rector, B. Dam, R. Griessen, Phys. Rev. Lett. 83, 2054 (1999).

[3] E. Baruch-El, M. Baziljevich, T.H. Johansen, Y. Yeshurun, J. Supercond. Nov. Magn. 28, 379 (2015).

[4] D.V. Kadygrob, N.M. Makarov, F. Pérez-Rodríguez, T.M. Slipchenko, V.A. Yampol'skii, New J. Phys. 15, 023040 (2013).

[5] C. Romero-Salazar, F. Pérez-Rodríguez, Supercond. Sci. Technol. 16, 1273 (2003); Appl. Phys. Lett. 83, 5256 (2003).

[6] I.F. Shampine, I. Gladwell, S. Thompson, Solving ODEs with MATLAB, Cambridge University Press, Cambridge 2003. 